Torsten Wilholt

\title{
Explaining Models \\ Theoretical and Phenomenological Models and their Role for the First Explanation of the Hydrogen Spectrum
}

\author{
[Manuscript version. The original publication appeared in \\ Foundations of Chemistry 7 (2), 2005, 149-169.]
}

\begin{abstract}
Traditional nomological accounts of scientific explanation have assumed that a good scientific explanation consists in the derivation of the explanandum's description from theory (plus antecedent conditions). But in more recent philosophy of science the adequacy of this approach has been challenged, because the relation between theory and phenomena in actual scientific practice turns out to be more intricate. This critique is here examined for an explanatory paradigm that was groundbreaking for 20th century physics and chemistry (and their interrelation): Bohr's first model of the atom and its explanatory relevance for the spectrum of hydrogen. First, the model itself is analysed with respect to the principles and assumptions that enter into its premises. Thereafter, the origin of the model's explanandum is investigated. It can be shown that the explained "phenomenon" is itself the product of a host of modelling accomplishments that stem from an experimental tradition related to 19th century chemistry, viz. spectroscopy. The relation between theory and phenomenon is thus mediated in a twofold way: by (Bohr's) theoretical model and a phenomenological model from spectroscopy. In the final section of the paper an account is outlined that nevertheless permits us to acknowledge this important physico-chemical achievement as a case of (nomological) explanation.
\end{abstract}

Within the philosophical analysis of scientific explanation, natural laws have traditionally played a prominent role. The traditional assumption is that a successful explanation of a phenomenon consists in the derivation of its description from at least one natural law and a set of antecedent conditions. The family of approaches starting from this assumption can be subsumed under the label 'nomological scheme of scientific explanation'. It has been subject to persistent criticism in the past decades. The predominant line of disagreement (concentrating primarily on the symmetry of explanation and prediction $^{1}$ ) has been maintaining that the sufficient criteria of the nomological scheme are too generous; that it admits instances which are in fact no explanations.

In this paper, however, I will concentrate on the more recent criticism purporting that the necessary criteria of the nomological scheme are too strict-taking them seriously, one would not find any explanations in the sciences at all. This line of criticism has primarily been put forward by Nancy Cartwright. According to her, the nomological scheme is something like a pious fiction that cannot be located in the reality of scientific practice. She suggests that the variform and erratic reality of 
experience can only by made describable and controllable in highly situative laboratory contexts. Phenomenological models serve to achieve this goal. These can in turn be brought into a mediated relation to theoretical laws, but a direct derivation of phenomena from laws is, according to Cartwright, out of the question: "[T]o explain a phenomenon is to construct a model which fits the phenomenon into a theory." (Cartwright, 1983, 17.) Laws do not have an explanatory function within this task:

[L]aws - in the conventional empiricist sense-have no fundamental role to play in scientific theory. In particular, scientific explanation seems to proceed entirely without them. They are the end-point of explanation and not the source. (Cartwright, 1989, 185.)

In this paper, Cartwright's thesis that the relation between theory and phenomenon is only fashioned by means of the mediation of models will be illustrated by a prominent example: I will undertake a close inspection of Bohr's first model of the hydrogen atom. However, I also intend to show that the laws of physical theory can, pace Cartwright's pessimism, explain natural phenomena with the aid of mediating models. Bohr's example is a good case in point that the use of models is not an indicator for the limited explanatory power of natural laws, but that models are a part of nomological explanations and extend their scope-if we base our considerations on a conception of nomological explanation that conforms to actual scientific practice.

\section{Bohr's model: elements of theory and antecedent conditions}

There is a very simple view of Bohr's first model of the hydrogen atom as published in $1913 .^{2}$ According to this view, Bohr assembled some new and many old elements of physical theory, postulated an inner structure of the hydrogen atom and derived, from all this, the frequencies of the spectral radiation of hydrogen. (Which therefore, according to the nomological scheme, also received an explanation.) But this rough sketch is rather a caricature. A more precise look at Bohr's actual proceeding brings new details into view. ${ }^{3}$

Let us first describe Bohr's model, beginning with the antecedent conditions:

- The antecedent conditions governing the arrangements of theoretical elements in Bohr's model follow the example of Ernest Rutherford's model. Electrons are orbiting a nucleus whose spatial extension is negligible if compared to the radius of the orbit. The positive electrical charge of the atom is concentrated in the nucleus, etc.

- Another important antecedent condition is the number of electrons. Bohr himself had shown, in a paper on the absorption of $\alpha$-particles, that the hydrogen atom possesses exactly one electron. (Cf. Mehra and Rechenberg, 1982, 185.) 
More decisive for the character of Bohr's model are the natural laws and theoretical principles that, according to Bohr, govern this arrangement of antecedent conditions. In what follows, I will refer to these collectively as 'elements of theory'.

- First, we have classical elements of theory like Newtonian mechanics and Coulomb's law. They describe the stationary states of the model. The electron can also be numbered among the classical elements of theory. Since J.J. Thomson's experiments, it had an allocated place in physical theory, including increasingly established values of its charge $e$ and mass $m$, which were conceived of as natural constants.

- But the classical elements are complemented by the quantum hypothesis, i.e. the theoretical assumption that electromagnetic radiation only exists in certain energy doses $h v$ depending in size on the frequency $v$ ( $h$ being Planck's constant), each of which is homogeneous with regard to frequency. Bohr had already arrived at the conviction that the classical theories were insufficient for the purposes of atomic physics in his dissertation on the physics of electrons in metals: (Cf. Heilbron und Kuhn, 1969, 213-223.) "The cause of failure is very likely this: that the electromagnetic theory does not agree with the real conditions in matter." (Cit. from Heilbron, 1985, 29.) The citation shows that for Bohr, the quantum hypothesis was not an ad hoc supposition, but a prerequisite for a suitable theoretical frame.

- An additional element of theory must be seen in Bohr's principle of correspondence. The theory now contained competing principles of electromagnetic radiation, one classical and one quantum-theoretical. The necessity emerged to prevent contradictions at the joint of their realms of application. The principle of correspondence serves this purpose. In its early form it essentially says that the radiation emitted (resp. absorbed) by the atom during changes between states of higher energy (i.e. states of large orbital radius and low orbital velocity) must approximate the one predicted by the classical theory of a charged oscillator. Bohr does not extract this principle from established theory, but establishes it as a new element of theory. It was to survive several changes of Bohr's atomic model and even the crisis of the older quantum theory in the 1920's. (Cf. Falkenburg, 1997, 34 f.)

We will now investigate how far elements of theory and antecedent conditions such as the ones indicated above can help us to determine all of the characteristics of Bohr's first model of the atom.

The hypothesis of quantum discontinuity serves to solve the notorious problems of Rutherford's model. The radiation of an electron spiralling towards the nucleus would be continuous and continuously changing in wavelength. Just this kind of radiation is prohibited, for atomic dimensions, by the quantum hypothesis. Therefore, Bohr's model must have stationary states with stable orbits. The task remains to settle just which states are the stationary ones. 


\section{Bohr's model: a decisive additional supposition}

In the transition from a state of energy $W$ to one of energy $W^{\prime}$, the atom must, according to the quantum hypothesis, emit an homogeneous dose of radiation with frequency $v$, where

$$
v=\frac{W}{h}-\frac{W^{\prime}}{h} .
$$

Bohr got acquainted with the efforts of researchers in spectroscopy to grasp the empirically determined spectral lines of hydrogen by means of a clear mathematical representation. The decisive success in this enterprise is generally attributed to Johann Jakob Balmer. In later years, Bohr declared (in an interview with Léon Rosenberg): "As soon as I saw Balmer's formula the whole thing was immediately clear to me." (Cit. from Mehra and Rechenberg, 1982, 188.) In fact, Bohr was starting from the following more general formula due to Janne Robert Rydberg:

$$
v=\frac{R_{\mathrm{H}} c}{n_{1}^{2}}-\frac{R_{\mathrm{H}} c}{n_{2}^{2}} .
$$

Here $c$ is the speed of light and $R_{\mathrm{H}}$ an empirical constant, the so-called Rydberg constant. Inserting pairs of natural numbers, $n_{1}<n_{2}$, the frequencies of the spectral radiation of hydrogen result.

Now Bohr makes a momentous supposition: $\mathrm{He}$ assumes that the formal similarity of equations (1) and (2) is rooted in a kinship in content. This is evidenced by the fact that he presumes that (2) represents the transition form the $n_{2}$-th state to the $n_{1}$-th. While for experimental physicists like Rydberg the numbers $n_{1}$ and $n_{2}$ had only been calculational devices without physical meaning, in Bohr's model they turn into ordinal numbers of stationary states.

On the basis of this supposition, the successful construction of the remaining determinants of Bohr's model ensues according to the following outline. (Cf. Bohr, 1981 [1914], [294].) Equating $W / h$ from (1) with $R_{\mathrm{H}} c / n^{2}$ from (2) yields the energy values for the stationary states. From these and from the classical laws for a point mass orbiting around a centre of force, values for the angular frequencies $\omega_{n}$ can be derived, such that $\omega_{n}$ only depends on the number $n$ of the state and some constants including the empirical Rydberg constant. This is important for the principle of correspondence which is supposed to warrant a smooth transition from the quantum-theoretical to the classical treatment of electromagnetic radiation. The classical treatment is based on the notion of a wave caused by an oscillator. If the orbiting electron is to impersonate this oscillator, then the orbital frequency $\omega_{n}$ must approximate the frequency $v$ of radiation in the transition between the $n$-th and the $(n+1)$-th state for large $n$. It turns out that exactly this happens if the Rydberg constant is calculated from the constants $e, m, c$ and $h$ according to 


$$
R_{\mathrm{H}}=\frac{2 \pi^{2} e^{4} m}{c h^{3}}
$$

One of the most important triumphs of Bohr's model of the atom is the excellent match of this value for $R_{\mathrm{H}}$ with the one empirically determined.

The fact that this triumph is ultimately based upon the identification of elements from the formulas (1) and (2) has prompted John Heilbron and Thomas Kuhn (1969, 70) to conclude that Rydberg's formula (2) is itself a premise of Bohr's model. But as we have seen, the value of $R_{\mathrm{H}}$ is a result of his theoretical considerations. What is presupposed by Bohr is merely the form of (2). Admittedly, this alone is noteworthy enough. We must diagnose that a supposition enters into Bohr's model that is neither justified by elements of theory nor by his chosen antecedent conditions. It is the assumption that the frequency of the radiation emitted (resp. absorbed) in the transition between the $n_{1}$-th stationary state and the $n_{2}$-th can be represented in a formula of the form

$$
v=C\left(\frac{1}{n_{1}^{2}}-\frac{1}{n_{2}^{2}}\right) \text {, with } C \text { a constant. }
$$

Assumptions that can be negatively characterised in just this way (i.e., as belonging neither to the elements of theory endorsed in the model nor to the antecedent conditions) often enter into processes of theoretical modelling. I will refer to them as "additional assumptions".

The mere presence of additional assumptions (that characteristically lack any kind of deductive or inductive support prior to the construction of the model) is apt to cast doubt upon the explanatory force of such a model. But we will return to that later. Leaving the question of explanatoriness aside for the moment, one could still be inclined to admit that Bohr has succeeded in theoretically modelling a natural phenomenon, viz. the phenomenon of the spectral radiation of hydrogen.

But there is another complication lurking here, urging us to concede a point to Nancy Cartwright. What the model arrives at, viz. Rydberg's formula for the spectral lines of atomic hydrogen, is itself a model. ${ }^{4}$ I will call this model a phenomenological model (as opposed to 'theoretical model', which applies to Bohr's own considerations). In the following section, I will outline some of the modelling achievements contained in this model. They have primarily been effected by 19th century experimental science.

\section{The phenomenological model of hydrogen}

In the mid-1850's, Gustav Kirchhoff and Robert Bunsen studied diverse substances by burning them in a colourless gas flame and decomposing the emitted light into its various coloured constituents. They confirmed that the emitted spectrum is 
characteristic for each chemical substance, and that the spectral lines of elementary substances also appear in the spectra of chemical composites containing them. Kirchhoff and Bunsen thereby established the spectrum as a fingerprint of the chemical element. $^{5}$

In the 1870's, interest increased among scientists to bring order into the as yet inscrutable tangle of lines of each individual spectrum. By and by, the spectrum of hydrogen became the preferred object of these efforts. Therefore, it became very desirable to certify a satisfactory number of sufficiently precise wavelengths of the hydrogen spectrum. For a long time, there were only four lines that could be unambiguously attributed to the hydrogen spectrum. A systematic study of the ultraviolet spectrum of hydrogen, published in 1879 by Hermann Wilhelm Vogel, marked an important progress in this enterprise. (Vogel, 1879a, 1879b.) Vogel, a chemist at the Technische Hochschule in Berlin-Charlottenburg, had constructed a specially developed apparatus, the "Spectrograph", to photograph the spectrum.

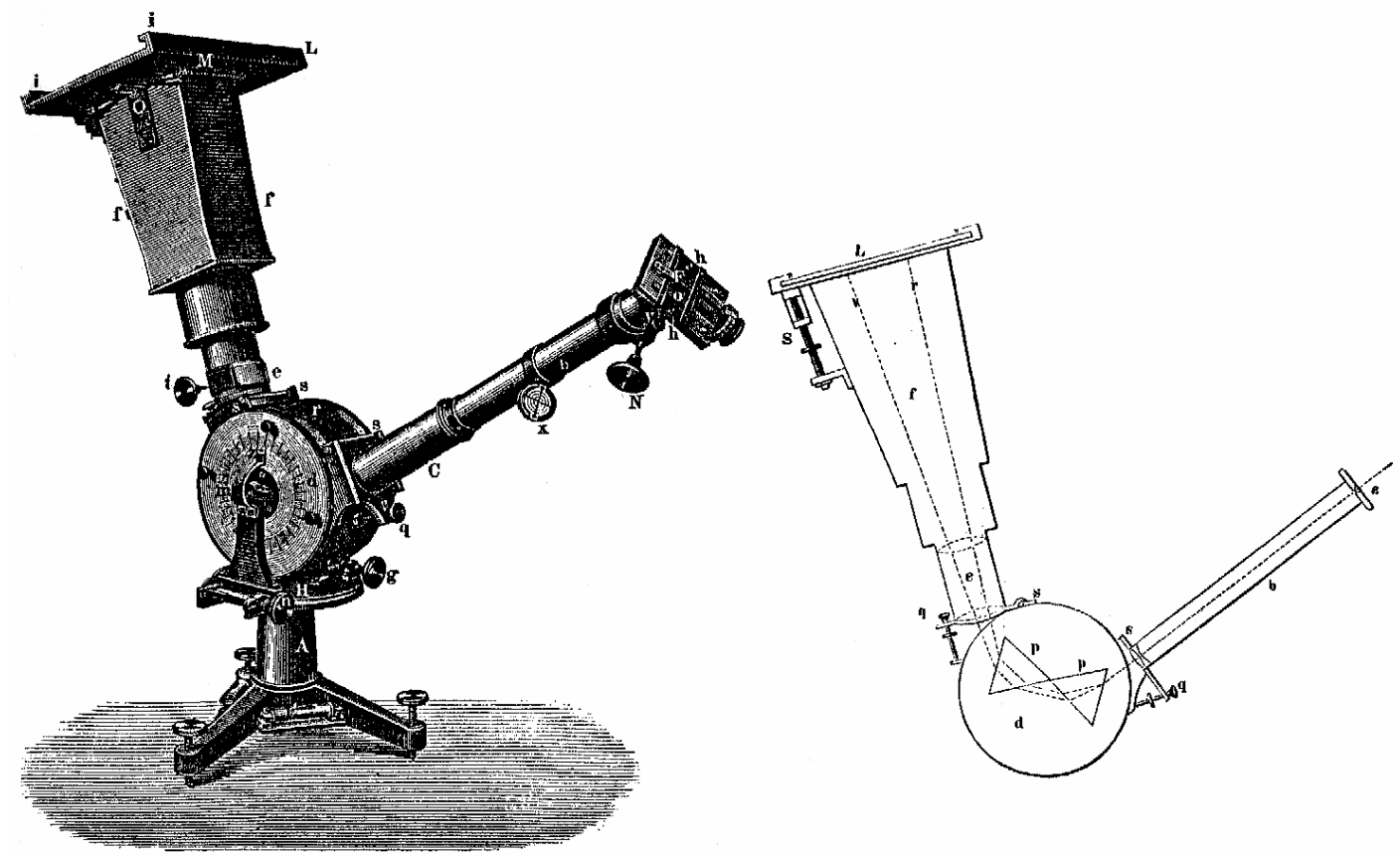

Vogel's “Grosser Spectrograph”. From Schellen, 1883, $438 \mathrm{f}$.

Special attention had to be paid to screen off disturbing light, because the ultraviolet part of the spectrum turned out to be extremely weak. For the same reason, Vogel used two flint glass prisms, since regular glass absorbs ultraviolet light in great proportions. And he projected the spectrum to newly developed gelatine dry plates, allowing a longer exposure than the traditional wet plates. (Cf. Vogel, 1879a, 116.)

All experimental circumspection could not eliminate one source of disturbance. The hydrogen was contained, under very low pressure, in glass tubes, so-called Geissler tubes, to which electrical voltage could be applied in order to stimulate the gas to glow. 
But during the production of the hydrogen, total purity could not be obtained. ${ }^{6}$ As a result, the photographs contained several lines known to belong to spectra of substances other than hydrogen. Some lines also conspicuously coincided with spectral lines of mercury. Mercury vacuum pumps were used to make the Geissler tubes. (One such impurity of the hydrogen spectrum in a Geissler tube proves to be inexpugnable even to this day: There are always some $\mathrm{H}_{2}$ molecules in the tube excited by the voltage, such that the spectrum of the $\mathrm{H}$ atom is invariably superposed by a molecular spectrum. ${ }^{7}$ )

As a result of all this, there had to be a decision based upon a certain effort of interpretation in order to determine which of the lines belong to the spectrum of hydrogen. A negative comparison had to be carried through with the known spectral lines of other substances that could reasonably be expected in the Geissler tubes, as well as a positive comparison with spectral decompositions of starlight in which a great hydrogen proportion was suspected. Vogel himself undertook a tentative alignment of the 32 wavelengths measured by him with a multitude of known values, but much had to be left open. He refrained from explicitly explicating individual new lines as hydrogen lines.

This attribution was only achieved with some definiteness by a discovery made by Johann Jakob Balmer in 1884. Balmer, a Swiss grammar school teacher with a penchant for number problems, was looking for a simple mathematical formula to account for all wavelengths of the hydrogen spectrum. By a method of trial and error, he found the following remarkably simple expression:

$$
\lambda=h \frac{m^{2}}{m^{2}-n^{2}}, \text { where } n=2 \text { and } h=3645,6 \cdot 10^{-10} \mathrm{~m} .
$$

Inserting the natural numbers from 3 to 10 for $m$, one gets wavelengths for the four visible hydrogen lines, as well as for four of Vogel's ultraviolet lines. The astronomer William Huggins had observed an additional line in the light of white stars, matching well with the value for $m=11 .^{8}$ 
a)
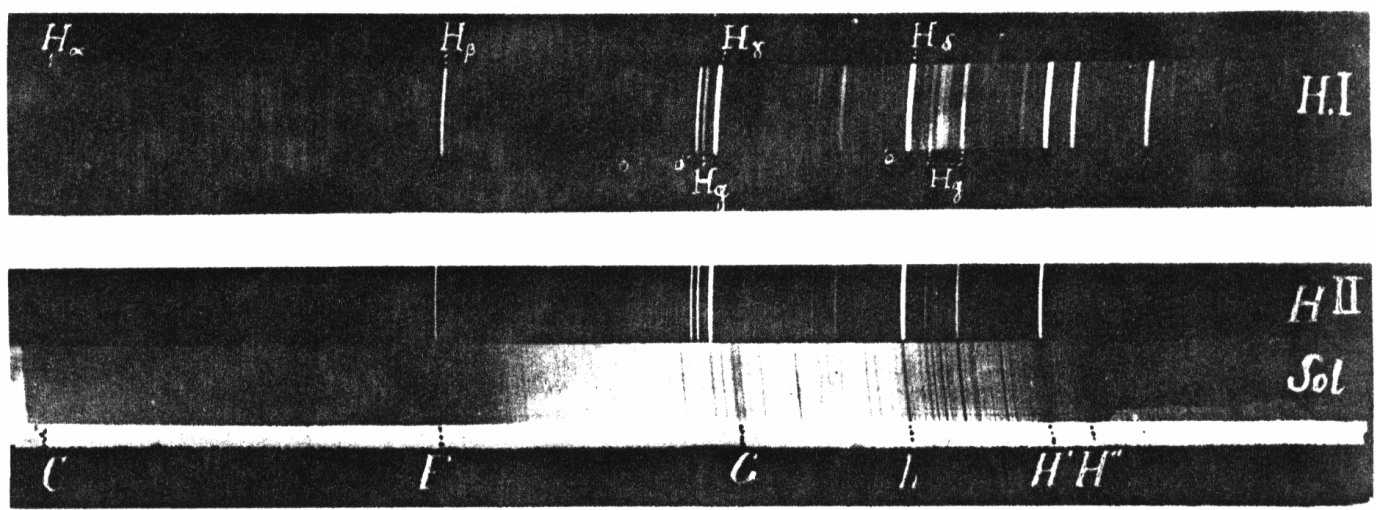

b)

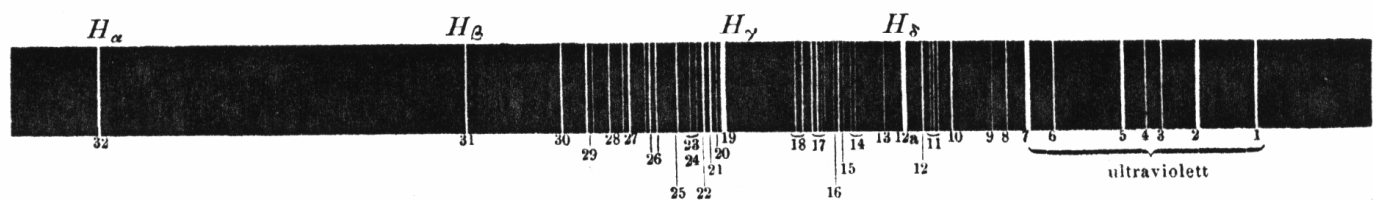

c)
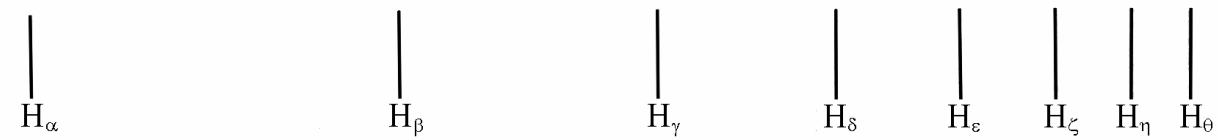

a) Two of Vogel's photographs of the hydrogen spectrum, with the spectrum of the sun for comparison

(supplement "Tafel I" from Vogel, 1879b).

b) Graphic representation of the lines measured in Vogel's experiments (from Vogel, 1879b, 589).

c) The selection effected by Balmer's formula.

With respect to the totality of Vogel's results, however, the selection effected by Balmer's formula seems utterly ad hoc. Weak lines are attributed to the hydrogen spectrum (like line no. 1), while other lines are neglected that had been classified by Vogel as "quite strong" or "stronger" and could not be attributed to any known substance (especially lines no. 6, 22 and 30).

Then what was the justification to accept the Balmer formula as a representation of the hydrogen spectrum? It was simply the notion that the spectrum had to be the product of a single physical system, which had spawned the expectation of a uniform mathematical representation. Already prior to Balmer's discovery, physicists had pondered the wavelengths and found it "conspicuous that they lie on, or very near, a definite curve, which would not happen by chance", as Gerorge Johnstone Stoney commented on Huggins' starlight spectra. He also concluded that the source of the radiation had to be "one physical system", possibly "some vibrating system (like those of an elastic rod or bell for example)". "In keeping with the same analogy, Arthur Schuster had announced to the BAAS in 1882 the ambition of spectroscopy "to find out the shape of a bell by means of the sounds which it is capable of sending out." Balmer's formula did not immediately suggest any conclusions about the nature of the 
radiation's source, it provided a mathematical regularity that could serve as the basis for further generalisations and research.

When some years later Janne Robert Rydberg and Walther Ritz developed generalisations of the Balmer formula, they were still working on the phenomenological surface. They arrived at the formula

$$
\frac{1}{\lambda}=R_{\mathrm{H}}\left(\frac{1}{n_{1}^{2}}-\frac{1}{n_{2}^{2}}\right),
$$

which, in the form of (2), was later to play the decisive role in Bohr's model. It was equivalent ${ }^{11}$ to Balmer's original version for $n_{1}=2$ and $n_{2}=3,4,5, \ldots$, but could also accommodate the infrared lines that Friedrich Paschen had discovered in 1908 (for $n_{1}=3$ und $\left.n_{2}=4,5,6, \ldots\right)$.

We see now that the Rydberg formula (6) is the end product of an elaborate process of material manipulation (purification of the hydrogen, inclusion in an apparatus designed to accentuate the sought-after ultraviolet lines) as well as conceptual manipulation (selection of the spectral lines that are supposed to belong to the hydrogen spectrum, representation by uniform mathematical expressions). Both kinds of manipulations have been highlighted as crucial elements of modelling by Ernan McMullin, who called them "causal idealization" and "construct idealization" (McMullin, 1985, 254-259, 264-268). I maintain that the result of these manipulations should be called a phenomenological model. This is not just because it contains idealisations-which alone makes it a model, but does not explain in which sense it is a phenomenological one.

What makes the representation a phenomenological one is, I suggest, that it was arrived at by means of a modelling process that started from measurements and observations, and received relatively little theoretical information on the way. This concept of phenomenological model partly reflects that the notion was first used in the philosophical discussion in order to label purely descriptive representations lacking any theoretical justification (cf., e.g., Cartwright et al., 1995). Margaret Morrison has criticised this way to distinguish between theoretical and phenomenological models (Morrison 1999). She argues that the models typically described as phenomenological are neither free of theory, nor are they the only ones to describe or represent reality-the so-called theoretical models can do so as well.

Though I think that both these points must be acknowledged, I still maintain that the distinction can be useful if it is used to distinguish models according to their provenance. While a theoretical model is assembled from elements of theory, a phenomenological model's history begins with an account of observed properties or behaviour, which is then transformed into a useful description. The modelling process takes a different direction in each case. 
The distinction may still not be clear-cut and exclusive in each and every case: There may be models that start at both ends and piece together elements of theory and phenomenological descriptions at the same time. But there are many cases where we can identify a phenomenological and a theoretical model in the sense above; and the first explanation of the hydrogen spectrum is one of them. ${ }^{12}$

When it is said that Niels Bohr explained the spectrum of hydrogen, what is claimed is usually that in his model the Rydberg formula $(2) /(6)$ can be derived. Yet the history of spectroscopy shows that it was never simply given what "the spectrum of hydrogen" is. Rather, the formula derived from Bohr's model is itself a (phenomenological) model.

\section{Bohr's model as a nomological explanation: outline of an altered scheme}

From the perspective of the traditional nomological scheme, these findings about Bohr's model cast severe doubts upon its explanatoriness for two reasons. The first is that nomological schemes do not admit additional suppositions as found in Bohr's theoretical model. Premises of an explanation must either be natural laws (replaced, in our analysis, by the more generous concept of elements of theory) or independently verifiable antecedent conditions. ${ }^{13}$ The second reason is that the theoretical model does not permit the derivation of a true and straightforward description of some given phenomenon, but only of an idealised, materially and conceptually manipulated replica of a phenomenon - a phenomenological model.

Observations like the characteristics of the modelling process that have been emphasised so far have prompted Nancy Cartwright to deny the explanatory force of natural laws altogether. However, I think that in spite of these historical findings it can still make sense to maintain that Bohr did explain the spectrum of hydrogen. (Of course, this judgement requires an historically qualified assessment of explanatory achievements. From a present day perspective, we wouldn't accept Bohr's model as an explanation for the simple reason that it makes presuppositions which we hold to be false-e.g., that electrons can do such things as describe orbits.)

To expose the explanatory merits of our case, it is useful to consider the possibility of an altered scheme of nomological explanations that takes into account the role of theoretical and phenomenological models in the explanatory process. (The scheme is in fact inspired by Cartwright's own "simulacrum account" of scientific explanation; Cf. Cartwright 1983, ch. 8. However, it is constructed not as an alternative to the nomological scheme, but as its refinement. Models must then be regarded not as disrupting the explanatory scope of laws, but as carrying it forward.) According to this altered scheme, to explain a phenomenon is to idealise it into a phenomenological model that can in turn be set into a relation of analogy to a theoretical model. The positive analogy must at least include those aspects of the phenomenological model that 
are supposed to receive an explanation. The corresponding theoretical model must be a concretisation from natural laws. That means that the theoretical model is an abstract structure collectively defined by some laws and some concretising conditions. The latter may include the usual antecedent conditions as well as additional suppositions as in Bohr's case, for the requirement of independent verifiability turns out to be too strict. But every model of scientific explanation will plausibly demand that the explanans itself can consistently be held true if it is supposed to explain anything. Therefore it should at the very least be possible to assume that the concretising conditions' claims about the system under discussions actually apply.

A few words should be said about the choice of the concept of analogy to characterise the relation between phenomenological and theoretical model. This concept's use within philosophy of science goes back to Mary Hesse (1963)—especially the notion of positive analogy, meaning the set of features that the two analogous systems are assumed to definitely share. Hesse spelled out analogy in terms of similarity (cf. Hesse, 1963, 66), so why not speak of similarity in the first place? I think the concept of analogy is to be preferred because it highlights the active part of the scientist that is often required in order to bring phenomenological and theoretical models together. Peter Achinstein described analogy as a relation that is proposed by a speaker who is asking "those for whom the analogy is intended to think of $X$ 's as $Y$ 's." (Achinstein, 1968, 207). In our case, the relevant analogy was to see the pattern of spectral lines (as represented in the Rydberg formula) as the radiation emitted by electrons falling into lower stationary states. It was Bohr himself who drew the analogy — as soon as he saw the formula, if we are to trust his own account of the story.

(Again, this is not to presume that some analogy is always required to bridge the phenomenological-theoretical gap in every case of scientific modelling. In some cases, where the modelling process is from the outset integrating theoretical and phenomenological considerations in one representation, the analogical step will become unnecessary. Morrison's example of Ludwig Prandtl's boundary layer model of fluid dynamics may be a case in point. Cf. Morrison, 1999, §3.4.2.)

Altogether, an explanation in accordance with the suggested scheme contains many hypothetical elements. The theoretical model emerges from the theoretical elements with the aid of concretising conditions, some of which might be hypothetical. Likewise, the phenomenological model will contain many creative elements, in its material as well as in its conceptual manipulations, that cannot be justified in every detail by appeal to observed facts or established theory. And finally, the analogy between the two models of different types always includes an hypothetical aspect, for it basically consists in the hypothetical assumption that the phenomenological model can be seen (i.e., described and explained) as a structure like the one specified in the theoretical model. 
Therefore, an explanation where the explanandum $P$ has the status of a necessary conclusion can only be found within the theoretical model. For the complete explanation, taking all mediating layers into account, cannot simply give the reasons that $P$ has/had to take place and nothing else. Instead, what it gives is roughly the following:

- Assuming that the phenomenological model $\Phi$ represents the phenomenon in good enough approximation ...

- ... and assuming that $\Phi$ can be understood $a s$ a system like the one specified in the theoretical model $\Theta$ (on the basis of an analogy) ...

- ... and assuming that the natural laws from theory $T$ do in fact manifest themselves in such a system in approximately the way that the concretising conditions from $\Theta$ suggest ...

... $P$ has/had to take place and nothing else.

Summing up, we can say that the suggested scheme only requires a nomological explanation to show that $\nabla_{e} \square_{n} P$. Here, the operator $\diamond_{e}$ symbolises possibility in terms of an epistemic modality, ${ }^{14}$ while $\square_{n}$ stands for the nomological necessity of the involved laws. A successful explanation would then demonstrate that it is epistemically possible that $P$ is nomologically necessary.

Therefore, let us pause a moment and ponder whether such an explanation can at all be satisfactory. The question can be posed in the following way: Can a satisfactory answer to a why-question take the form $\diamond_{e} \square_{n} P$ ?

We know from our everyday experience with the language game pertaining to questions of the form "Why $P$ ?" that an answer to such a question need not necessarily consist of the specification of secured knowledge that entails $P$. Often, a satisfactory answer takes the form of a possible story according to which $P$ had to take place and nothing else. A general kind of example for this is provided by certain typical probabilistic explanations that go along the following lines: " $P$ took place because under the given circumstances there is an increased probability for such and such processes to occur." The explanation in these cases suggests a story that is probable, though not certain (viz., that the processes in question did indeed occur) and according to which $P$ had to take place. (As is well known, such explanations can even succeed when the probabilities in question are low, as in the notorious example: "Why does $X$ suffer from paresis? - Given his history of untreated latent syphilis, there is a known, non-zero probability of certain physiological processes to occur that inevitably lead to paresis.”)

Other examples can be found in many evolutionary explanations of questions like "Why do these organisms have this puzzling trait?" Often, the answer consists in a story that details how, if the biological kind in question has evolved under certain specified 
circumstances for an extended period of time, that accounts for just the set of traits that were found perplexing before. Again, the story must be possible, but need not be conclusively verified by a corpus of secured knowledge for the explanation to be satisfactory.

The proof that it is epistemically possible that $P$ is nomologically necessary can be just as satisfactory as these familiar kinds of explanation that tell or suggest a possible story according to which $P$ had to take place, because in the end it does just the same. (In this sense, we can understand the Bohr model, together with the phenomenological modelling achievements of spectroscopy, as producing a possible story according to which the hydrogen atom has to emit just the kind of spectral radiation that it in fact does emit.) This is why nomological explanations according to the liberalised scheme can give satisfactory answers to scientific why-questions.

The approach to regard scientific explanations as answers to scientific whyquestions was set off mainly by Bas van Fraassen (1980, ch. 5). ${ }^{15}$ His pragmatic theory was severely criticised because he had forborne from determining what kinds of relation qualify as the "relevance relation" in which the explanans should stand to the explanandum (and its contrast class) and had therefore, according to his critics, rendered the notion of explanation arbitrary. (Cf. Kitcher and Salmon 1987.) ${ }^{16}$ No such arbitrariness befalls my suggestions in this section. For the scheme that I am proposing, though liberalised, is much more traditional than van Fraassen's; it is a scheme for nomological explanations and should not be considered a pragmatic approach. The relevance relation at the core of each explanation is nomological necessitation. Although enclosed in a big epistemic "possibly", it is always nomological necessitation that carries the explanatory force.

In the 1960's, some philosophers found it important to note that not every explanation need be an answer to a why-question, and that some are answers to howpossibly-questions instead. (Cf. Hempel 1965, 428-430.) In terms of this distinction, it might be remonstrated that explanations in accordance with the suggested liberalised scheme of nomological explanation provide answers to how-possibly-questions rather than why-questions. I see no reason to object. In fact I find it suitable to say that Bohr's explanatory achievement was to answer the question: "How can it possibly be that the radiation emitted by hydrogen atoms always takes the form of the characteristic spectrum we are observing?"

To conclude, it is two things that all these considerations taken together point to. Firstly, the thesis that there can be no question of an immediate deductive relation between the elements of theory and the description of the phenomenon is neatly reproducible for the prominent example of Bohr's first model of the hydrogen atom. But Bohr's theoretical model and the spectroscopic phenomenological model succeed in mediating between the theoretical level and the phenomena. ${ }^{17}$ In doing so, Bohr's model presupposes a peculiar ensemble of elements of theory and extends its explanatory force 
to cover an heretofore unexplained phenomenon. The second lesson I propose to draw from this is therefore the following: If we liberalise our demands on nomological explanations, we do not need to abandon natural laws' claim to explanation.

\section{Notes}

1 The nomological scheme automatically turns every prediction from natural laws into an explanation. In many cases, this contradicts our intuitive judgements on what constitutes an explanation. The notorious problem example is the "explanation" of the length of a flag pole by means of the length of its shadow and the position of the sun.

2 See, e.g., McMullin, 1968, esp. 393.

3 A most precise look can be found in the many excellent analyses of Bohr's model undertaken by historians of science. See esp. Heilbron and Kuhn, 1969, Heilbron, 1977, Mehra and Rechenberg, 1982, chapter II and Darrigol, 1992, chapters 5 \& 6.

4 Cf. Cartwright, 1989, 207: "For the end-point of theory-licensed concretization is always a law true just in a model."

5 A thorough treatment of the history of spectroscopy can be found esp. in McGucken, 1969 and Mehra and Rechenberg, 1982, 156-168.

6 Vogel experimented with different procedures, cf. Vogel, 1879b, 587.

7 Cf., e.g., Haken and Wolf, 1993, $102 \mathrm{f}$.

8 Cf. Balmer, 1885, 83. Different values for $h$ had to be assumed to accommodate the measurements of each respective experimenter, but the deviations lay within tolerable limits.

9 In a letter to Huggins, as quoted in McGucken 1969, $120 \mathrm{f}$.

10 In a report on 'The Genesis of Spectra', as quoted in Mehra and Rechenberg 1952, $161 \mathrm{f}$.

11 Assuming the relation $R_{\mathrm{H}}=4 / h$ to Balmer's constant $h$.

12 It does no harm that the core representation of the phenomenological model, (6), is a formula that was later also derived within a theoretical model. What is decisive is that it was first arrived at by a process of idealisation and generalisation that started from measurements and observations and hardly received any theoretical input (with the possible exception of the very abstract notion that there should be a uniform mathematical representation for all the wavelengths).

13 Independent from the verification of the explanandum, that is. In the ancestor of all nomological schemes, Hempel and Oppenheim's deductive-nomological model, this is called for by the following condition: "[The theory] $T$ must be compatible with at least one class of basic sentences which has [the statement of antecedent conditions] 
$C$ but not [the explanandum] $E$ as a consequence." (Hempel und Oppenheim, 1953 [1948], 346.)

$14 \vartheta_{e}$ should roughly be interpreted as "possible according to best contemporary knowledge, i.e. permitted by established theory and not incompatible with available observational evidence".

15 Of course, van Fraassen did have important precursors, notably Silvain Bromberger (1966).

${ }^{16}$ It has, however, been put forward in defence of van Fraassen that he considers the choice of relevance relations restricted by the accepted scientific theories at the time. (Cf. Lloyd and Anderson, 1993 and van Fraassen, 1980, 126.)

17 These observations therefore fit in with the recent efforts of Margaret Morrison and others to establish the role of scientific models in general as one of mediators. See Morgan and Morrison (Eds), 1999.

\section{References}

P. Achinstein. Concepts of Science: A Philosophical Analysis. Baltimore \& London: Johns Hopkins Press, 1968.

J.J. Balmer. Notiz über die Spectrallinien des Wasserstoffs. Annalen der Physik und Chemie N.F. 25, pp. 80-87, 1885.

N. Bohr. On the Constitution of Atoms and Molecules [I]. In: U. Hoyer (Ed), Niels Bohr: Collected Works, vol. 2, pp. [161]-[185]. Amsterdam etc.: North Holland, 1981 [1913].

N. Bohr. On the Spectrum of Hydrogen. In: U. Hoyer (Ed), Niels Bohr: Collected Works, vol. 2, pp. [283]-[301]. Amsterdam etc.: North Holland 1981 [1914].

S. Bromberger. Why-Questions. In: R. G. Colodny (Ed), Mind and Cosmos, pp. 86-108. Pittsburgh: University of Pittsburgh Press, 1966.

N. Cartwright. How the Laws of Physics Lie. Oxford: Oxford University Press, 1983.

N. Cartwright. Nature's Capacities and their Measurement. Oxford: Clarendon Press, 1989.

N. Cartwright, T. Shomar and M. Suárez: The Toolbox of Science: Tools for the Buildings of Models with a Superconductivity Example. In: W.H. Herfel et al. (Eds), Theories and Models in Scientific Process, (= Poznan Studies in the Philosophy of the Sciences and the Humanities, vol. 44), pp. 137-149, Amsterdam \& Atlanta: Rodopi, 1995.

O. Darrigol. From c-Numbers to q-Numbers: The Classical Analogy in the History of Quantum Theory. Berkeley etc.: University of California Press, 1992. 
B. Falkenburg. Modelle, Korrespondenz und Vereinheitlichung in der Physik. In: B. Falkenburg and S. Hauser (Eds), Modelldenken in den Wissenschaften (= Dialektik 1997/1), pp. 27-42. Hamburg: Meiner, 1997.

H. Haken and H.C. Wolf. Atom- und Quantenphysik: Einführung in die experimentellen und theoretischen Grundlagen. 5th ed. Berlin, New York etc.: Springer, 1993.

J.L. Heilbron. Lectures on the History of Atomic Physics 1900-1922. In: C. Weiner (Ed), History of Twentieth Century Physics, $(=$ Rendiconti della scuola internazionale di fisica "Enrico Fermi" 57), pp. 40-108. New York \& London: Academic Press, 1977.

J.L. Heilbron. Bohr's First Theories of the Atom. Physics Today 38 (10), pp. 28-36, 1985.

J.L. Heilbron and T.S. Kuhn. The Genesis of the Bohr Atom. Historical Studies in the Physical Sciences 1, pp. 211-290, 1969.

C.G. Hempel. Aspects of Scientific Explanation and Other Essays in the Philosophy of Science. New York: The Free Press, 1965.

C.G. Hempel and P. Oppenheim. The Logic of Explanation. In: H. Feigl and M. Brodbeck (Eds), Readings in the Philosophy of Science, pp. 319-352. New York: Appleton-Century-Crofts, 1953 [1948].

M.B. Hesse. Models and Analogies in Science. London \& New York: Sheed and Ward, 1963.

P. Kitcher and W. Salmon. Van Fraassen on Explanation. The Journal of Philosophy 48 (6), pp. 315-330, 1987.

E.A. Lloyd and C.G. Anderson. Empiricism, Objectivity and Explanation. Midwest Studies in Philosophy 18, pp. 121-131, 1993.

W. McGucken. Nineteenth-Century Spectroscopy: Development of the Understanding of Spectra 1802-1897. Baltimore \& London: Johns Hopkins Press, 1969.

E. McMullin. What Do Physical Models Tell Us? In: B. van Rootselaar and J.F. Staal (Eds), Logic, Methodology and Philosophy of Science III, pp. 385-396. Amsterdam: North-Holland, 1968.

E. McMullin. Galilean Idealization. Studies in History and Philosophy of Science 16 (3), pp. 247-273, 1985.

J. Mehra and H. Rechenberg. The Historical Development of Quantum Theory, vol. 1, part 1. New York, Berlin etc.: Springer, 1982.

M.S. Morgan and M. Morrison (Eds). Models as Mediators: Perspectives on Natural and Social Science. Cambridge: Cambridge University Press, 1999. 
M. Morrison. Models as Autonomous Agents. In: Morgan and Morrison (Eds), 1999, pp. 38-65.

H. Schellen. Die Spectralanalyse in ihrer Anwendung auf die Stoffe der Erde und die Natur der Himmelskörper, vol. 1, 3rd ed. Braunschweig: Westermann, 1883.

B. van Fraassen. The Scientific Image. Oxford: Clarendon Press, 1980.

H.W. Vogel. Über die photographische Aufnahme von Spectren der in Geisslerröhren eingeschlossenen Gase. Monatsberichte der königlich preussischen Akademie der Wissenschaften zu Berlin, pp. 115-119, 1879a.

H.W. Vogel. Über die Spectra des Wasserstoffs, Quecksilbers und Stickstoffs. Monatsberichte der königlich preussischen Akademie der Wissenschaften zu Berlin, pp. 586-604, $1879 \mathrm{~b}$. 